\title{
Angle and Time of Arrival Statistics for Circular and Elliptical Scattering Models
}

\author{
Richard B. Ertel and Jeffrey H. Reed, Senior Member, IEEE
}

\begin{abstract}
With the introduction of antenna array systems into wireless communication networks comes the need to better understand the spatial characteristics of the channel. Scattering models provide both angle of arrival (AOA) and time of arrival (TOA) statistics of the channel. A number of different scattering models have been proposed in the literature including elliptical and circular models. These models assume that scatterers lie within an elliptical and circular region in space, respectively. In this paper, the joint TOA/AOA, the marginal TOA, and the marginal AOA probability density functions (pdf's) are derived for the elliptical and circular scattering models. These pdf's provide insight into the properties of the spatial wireless channel.
\end{abstract}

Index Terms-Antenna arrays, propagation.

\section{INTRODUCTION}

$\mathbf{F}$ OR beamforming and emitter localization applications in which an array of sensors is used to receive communication signals, it is necessary to have channel models that provide angle of arrival (AOA) and time of arrival (TOA) information about the multipath components. To meet this challenge, geometrically based single bounce channel models have been proposed. These models assume that scatterers are randomly located in two-dimensional space according to a joint probability density function (pdf). For convenience, this joint pdf will be referred to as the scatterer density function or just scatterer density. From the assumed scatterer density, it is possible to derive the joint TOA/AOA, marginal AOA, and marginal TOA pdf's of the multipath components.

Various scattering models have been proposed in the literature [1]-[6]. Here we consider only the circular and elliptical models described in [1] and [2], respectively. The first assumes a uniform pdf for the position of scatterers inside an ellipse, in which the base station and the mobile are located at the foci [2]. The model was proposed for microcell environments where multipath components may originate near both the mobile and the base station. The second model assumes a uniform pdf for the position of scatterers within a radius about the mobile [1]. Such a scatterer density is more appropriate in macrocell environments in which antenna heights are typically higher than surrounding scatterers, making it less likely for multipath reflections to occur near the base station.

In this paper, the joint TOA/AOA pdf, marginal AOA pdf, and marginal TOA pdf at both the base station and mobile are

Manuscript received April 10, 1998; revised November 11, 1998.

The authors are with the Mobile and Portable Radio Research Group-432 NEB, Bradley Department of Electrical and Computer Engineering, Virginia Polytechnic and State University, Blacksburg, VA 24061 USA.

Publisher Item Identifier S 0733-8716(99)08959-3.

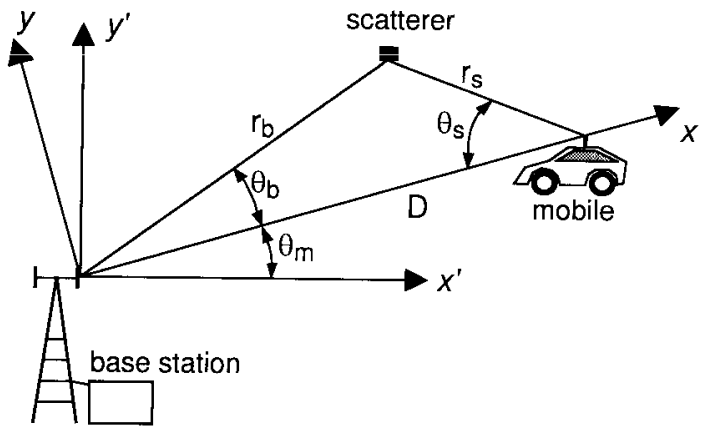

Fig. 1. Scatterer geometry.

derived for the elliptical and circular scattering models. The pdf's for the elliptical model were previously derived in [7]. Here a more general approach is used that allows the pdf's for both models to be derived using a common approach. The joint TOA/AOA pdf and the marginal TOA pdf for the circular model are original contributions of this paper.

\section{Problem Geometry And Assumptions}

Fig. 1 shows the geometry and notation used to derive the TOA and AOA pdf's of the scattering models. The mobile is separated from the base station by the distance $D$. Although only one scatterer is shown in the figure, it is assumed that there are many scatterers, the locations of which are described by the statistical scatterer density function $f_{x, y}(x, y)$. The results derived here apply to the ensemble of randomly located scatterers and make no assumptions regarding the number of scatterers present.

It is assumed that the effective antenna patterns are omnidirectional for both transmit and receive. In practice, the derived pdf's should be used in conjunction with knowledge of the actual antenna radiation patterns. The following additional assumptions are common to both the elliptical and circular models [1].

1) The signals received at the base station are plane waves which propagate in the horizontal plane.

2) Scatterers are treated as omnidirectional reradiating elements.

3) The signals that are received at the base station have interacted with only a single scatterer in the channel.

\section{JOINT TOA/AOA PDF'S}

We first derive the most general joint pdf's and then show how these pdf's simplify for particular scatterer densities. Let 
the $x y$ coordinate system be defined such that the base station is at the origin and the mobile lies on the $x$ axis, as shown in Fig. 1. It will be useful to express the scatterer density function with respect to the polar coordinates $\left(r_{b}, \theta_{b}\right)$ as an intermediate step in deriving the joint TOA/AOA pdf. The polar coordinates are related to the rectangular coordinates via the following set of equations

$$
\begin{aligned}
r_{b} & =\sqrt{x^{2}+y^{2}} \\
\theta_{b} & =\operatorname{atan}\left(\frac{y}{x}\right) \\
x & =r_{b} \cos \left(\theta_{b}\right) \\
y & =r_{b} \sin \left(\theta_{b}\right)
\end{aligned}
$$

where $(x, y)$ denotes the location of the scatterer.

The joint pdf $f_{r_{b}, \theta_{b}}\left(r_{b}, \theta_{b}\right)$ is found using [8]

$$
f_{r_{b}, \theta_{b}}\left(r_{b}, \theta_{b}\right)=\left.\frac{f_{x, y}(x, y)}{|J(x, y)|}\right|_{\substack{x=r_{b} \cos \left(\theta_{b}\right) \\ y=r_{b} \sin \left(\theta_{b}\right)}}
$$

where $J\left(r_{s}, \theta_{s}\right)$ is the Jacobian transformation given by

$$
\begin{aligned}
J(x, y) & =\left|\begin{array}{ll}
\frac{\partial x}{\partial r_{b}} & \frac{\partial x}{\partial \theta_{b}} \\
\frac{\partial y}{\partial r_{b}} & \frac{\partial y}{\partial \theta_{b}}
\end{array}\right|^{-1} \\
& =\left|\begin{array}{lr}
\cos \left(\theta_{b}\right) & -r_{b} \sin \left(\theta_{b}\right) \\
\sin \left(\theta_{b}\right) & r_{b} \cos \left(\theta_{b}\right)
\end{array}\right|^{-1}=\frac{1}{r_{b}} .
\end{aligned}
$$

Substituting (6) into (5) gives

$$
f_{r_{b}, \theta_{b}}\left(r_{b}, \theta_{b}\right)=\left|r_{b}\right| f_{x, y}\left(r_{b} \cos \left(\theta_{b}\right), r_{b} \sin \left(\theta_{b}\right)\right) \text {. }
$$

If we restrict $r_{b}$ to be positive, then (7) becomes

$$
f_{r_{b}, \theta_{b}}\left(r_{b}, \theta_{b}\right)=r_{b} f_{x, y}\left(r_{b} \cos \left(\theta_{b}\right), r_{b} \sin \left(\theta_{b}\right)\right) \text {. }
$$

The next step is to find a relationship between $r_{b}$ and the delay $\tau$ of the multipath component. Applying the law of cosines to the triangle shown in Fig. 1 gives

$$
r_{s}^{2}=D^{2}+r_{b}^{2}-2 r_{b} D \cos \left(\theta_{b}\right) .
$$

The total path propagation delay is given by

$$
\begin{aligned}
\tau & =\frac{r_{b}+r_{s}}{c} \\
& =\frac{1}{c}\left(r_{b}+\sqrt{D^{2}+r_{b}^{2}-2 r_{b} D \cos \left(\theta_{b}\right)}\right) .
\end{aligned}
$$

Squaring both sides of (10) and solving for $r_{b}$ results in

$$
r_{b}=\frac{D^{2}-\tau^{2} c^{2}}{2\left(D \cos \left(\theta_{b}\right)-\tau c\right)}
$$

The joint TOA/AOA pdf is given by

$$
f_{\tau, \theta_{b}}\left(\tau, \theta_{b}\right)=\left.\frac{f_{r_{b}, \theta_{b}}\left(r_{b}, \theta_{b}\right)}{\left|J\left(r_{b}, \theta_{b}\right)\right|}\right|_{r_{b}=\left(D^{2}-\tau^{2} c^{2} / 2\left(D \cos \left(\theta_{b}\right)-\tau c\right)\right)}
$$

where $J\left(r_{b}, \theta_{b}\right)$ is the Jacobian transformation

$$
\begin{aligned}
J\left(r_{b}, \theta_{b}\right) & =\left|\frac{\partial r_{b}}{\partial \tau}\right|^{-1} \\
& =\frac{2\left(D \cos \left(\theta_{b}\right)-\tau c\right)^{2}}{\left.D^{2} c+\tau^{2} c^{3}-2 \tau c^{2} D \cos \left(\theta_{b}\right)\right)} .
\end{aligned}
$$

Substituting (13) into (12) gives

$$
\begin{aligned}
f_{\tau, \theta_{b}}\left(\tau, \theta_{b}\right)= & \frac{D^{2} c+\tau^{2} c^{3}-2 \tau c^{2} D \cos \left(\theta_{b}\right)}{2\left(D \cos \left(\theta_{b}\right)-\tau c\right)^{2}} \\
& \cdot f_{r_{b}, \theta_{b}}\left(\frac{D^{2}-\tau^{2} c^{2}}{2\left(D \cos \left(\theta_{b}\right)-\tau c\right)}, \theta_{b}\right) .
\end{aligned}
$$

Next, we may express the joint TOA/AOA density in terms of the original scatterer density function using (8) and (11). The resulting joint pdf becomes

$$
\begin{aligned}
f_{\tau, \theta_{b}}\left(\tau, \theta_{b}\right)= & \frac{\left(D^{2}-\tau^{2} c^{2}\right)\left(D^{2} c+\tau^{2} c^{3}-2 \tau c^{2} D \cos \left(\theta_{b}\right)\right)}{4\left(D \cos \left(\theta_{b}\right)-\tau c\right)^{3}} \\
& \cdot f_{x, y}\left(r_{b} \cos \left(\theta_{b}\right), r_{b} \sin \left(\theta_{b}\right)\right)
\end{aligned}
$$

where $r_{b}$ is given by (11). Equation (15) expresses the joint TOA/AOA observed at the base station in terms of an arbitrary scatterer density.

When the scatterers are uniformly distributed within an arbitrarily shaped region $R_{A}$ with an area $A$, then the scatterer density function is given by

$$
f_{x, y}(x, y)= \begin{cases}\frac{1}{A}, & x \text { and } y \in R_{A} \\ 0, & \text { else. }\end{cases}
$$

In this special case, the joint TOA/AOA pdf reduces to

$$
f_{\tau, \theta_{b}}\left(\tau, \theta_{b}\right)=\frac{\left(D^{2}-\tau^{2} c^{2}\right)\left(D^{2} c+\tau^{2} c^{3}-2 \tau c^{2} D \cos \left(\theta_{b}\right)\right)}{4 A\left(D \cos \left(\theta_{b}\right)-\tau c\right)^{3}}
$$

where the appropriate range of $\tau$ and $\theta_{b}$ are assumed.

Equation (17) gives the joint TOA/AOA pdf observed at the base station. Due to the symmetry of the geometry shown in Fig. 1, the joint TOA/AOA pdf at the mobile will have the same form as the TOA/AOA pdf observed at the base station. The only difference between the two is the range of $\tau$ and $\theta_{b}$ where the pdf is nonzero.

The relationship between $r_{b}$ and $\tau$ is identical in form to the relationship between $r_{s}$ and $\tau$, namely

$$
r_{s}=\frac{D^{2}-\tau^{2} c^{2}}{2\left(D \cos \left(\theta_{s}\right)-\tau c\right)}
$$

Repeating the derivation with respect to the polar coordinates at the mobile gives

$$
\begin{array}{r}
f_{\tau, \theta_{s}}\left(\tau, \theta_{s}\right) \\
=\frac{\left(D^{2}-\tau^{2} c^{2}\right)\left(D^{2} c+\tau^{2} c^{3}-2 \tau c^{2} D \cos \left(\theta_{s}\right)\right)}{4 A\left(D \cos \left(\theta_{s}\right)-\tau c\right)^{3}} .
\end{array}
$$

Note, however, that the range of $\theta_{b}$ and $\tau$ for which (17) is valid will be different from the range over which $\theta_{s}$ and $\tau$ is valid in (19). 


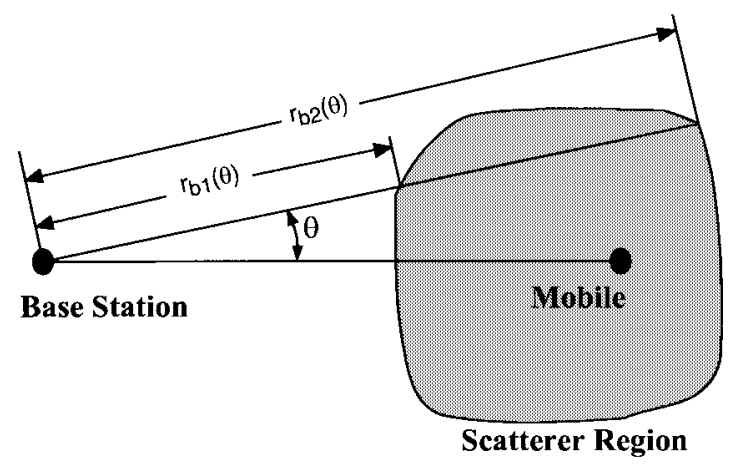

Fig. 2. Calculating AOA pdf.

\section{MARGINAL PDF'S}

\section{A. AOA Probability Density Function}

Although the AOA pdf could possibly be found by integrating the joint TOA/AOA pdf over $\tau$, a more straightforward approach is to integrate the polar coordinate system representation of the scatterer density function given in (8) with respect to $r_{b}$ over the range $r_{b 1}\left(\theta_{b}\right)$ to $r_{b 2}\left(\theta_{b}\right)$

$$
\begin{aligned}
f_{\theta_{b}}\left(\theta_{b}\right) & =\int_{r_{b 1}\left(\theta_{b}\right)}^{r_{b 2}\left(\theta_{b}\right)} f_{r_{b}, \theta_{b}}\left(r_{b}, \theta_{b}\right) d r_{b} \\
& =\int_{r_{b 1}\left(\theta_{b}\right)}^{r_{b 2}\left(\theta_{b}\right)} r_{b} f_{x, y}\left(r_{b} \cos \left(\theta_{b}\right), r_{b} \sin \left(\theta_{b}\right)\right) d r_{b} .
\end{aligned}
$$

Fig. 2 shows the definitions of $r_{b 1}\left(\theta_{b}\right)$ and $r_{b 2}\left(\theta_{b}\right)$ for an arbitrary scatterer region. For a uniform scatterer density with $f_{x, y}(x, y)=(1 / A)$

$$
\begin{aligned}
f_{\theta_{b}}\left(\theta_{b}\right) & =\int_{r_{b 1}\left(\theta_{b}\right)}^{r_{b 2}\left(\theta_{b}\right)} \frac{1}{A} r_{b} d r_{b} \\
& =\frac{1}{2 A}\left(r_{b 2}^{2}\left(\theta_{b}\right)-r_{b 1}^{2}\left(\theta_{b}\right)\right) .
\end{aligned}
$$

The functions $r_{b 1}\left(\theta_{b}\right)$ and $r_{b 2}\left(\theta_{b}\right)$ may be obtained from the polar coordinate representations of the boundary of the scatterer region. When the base station is located inside of the scatterer region, $r_{b 1}\left(\theta_{b}\right)=0$ and the AOA pdf reduces to

$$
f_{\theta_{b}}\left(\theta_{b}\right)=\frac{1}{2 A} r_{b 2}^{2}\left(\theta_{b}\right) \text {. }
$$

The same equations apply at the mobile when the scatterer density is referred to the polar coordinate system $\left(r_{s}, \theta_{s}\right)$ defined at the mobile (see Fig. 1). Hence, the AOA pdf at the mobile is given by

$$
f_{\theta_{s}}\left(\theta_{s}\right)=\frac{1}{2 A}\left(r_{s 2}^{2}\left(\theta_{s}\right)-r_{s 1}^{2}\left(\theta_{s}\right)\right)
$$

where the functions $r_{s 2}\left(\theta_{s}\right)$ and $r_{s 1}\left(\theta_{s}\right)$ define the boundary of the scatterer region. When the mobile is located inside of the scatterer region, $r_{s 1}\left(\theta_{s}\right)=0$, and the AOA pdf at the mobile becomes

$$
f_{\theta_{s}}\left(\theta_{s}\right)=\frac{1}{2 A} r_{s 2}^{2}\left(\theta_{s}\right) \text {. }
$$

These results will be used to find the AOA pdf's for the elliptical and circular scattering models in the corresponding sections that follow.

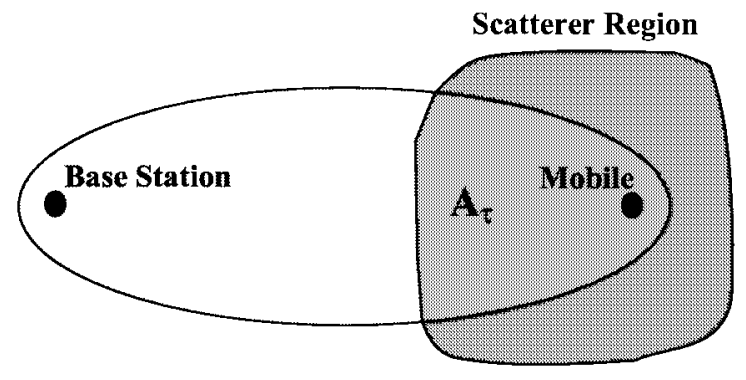

Fig. 3. Calculating TOA cumulative distribution function.

\section{B. $T O A p d f$}

Deriving a general TOA pdf for an arbitrary scatterer density function is more difficult. Integrating the joint TOA/AOA pdf over AOA, even for the case of a uniform scatterer density function, is nearly intractable and does not yield manageable results. A second, more promising approach is to first derive the TOA cumulative distribution function (cdf) and then take the derivative with respect to $\tau$ to obtain the desired TOA pdf. The TOA cdf may be calculated as the probability of a scatterer being placed inside the ellipse corresponding to a delay equal to $\tau$. The area of overlap of the ellipse with the scatterer region is illustrated in Fig. 3. In general, calculating the probability of a scatterer being placed inside the ellipse would involve a double integral of the scatterer density function over the overlap region.

For the case of a uniform scatterer density function, the TOA cdf is simply

$$
F(\tau)=\frac{A_{\tau}(\tau)}{A}
$$

Taking the derivative with respect to $\tau$ gives the desired TOA pdf

$$
f_{\tau}(\tau)=\frac{1}{A} \frac{d}{d \tau}\left(A_{\tau}(\tau)\right)
$$

The function $A_{\tau}(\tau)$ and the approach used to find this area are dependent upon the particular scatterer density function.

The TOA pdf observed at the base station is exactly the same as the TOA pdf seen at the mobile, since the distance traveled by a multipath component between the mobile and the base station by way of a scatterer is independent of the perspective.

\section{Elliptical SCATtering Model}

The assumed geometry for the elliptical scattering model (Liberti's model) is shown in Fig. 4 [2], [7]. Scatterers are uniformly distributed inside the ellipse with foci at the base station and mobile. The major axis of the ellipse is given by $c \tau_{m}$, where $\tau_{m}$ is the maximum delay associated with scatterers within the ellipse, and $c$ is the speed of light. The model is appropriate for microcell environments where antenna heights are relatively low. With low antennas, the base station may receive multipath reflections from locations near the base station as well as around the mobile. The elliptical model has the physical interpretation that only multipath components that arrive with a maximum delay of $\tau_{m}$ are 


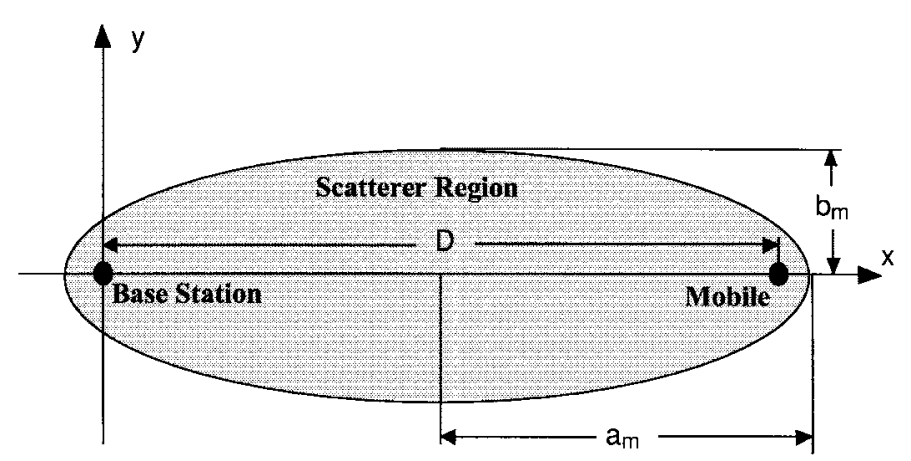

Fig. 4. Elliptical scatterer density geometry.

considered. Ignoring paths with longer delays may be justified, since such signal components will experience greater path loss and hence will have relatively low power compared to those with shorter delays. Therefore, provided that $\tau_{m}$ is chosen sufficiently large, nearly all of the power of the multipath signals of a physical channel will be accounted for by the model.

The parameters $a_{m}$ and $b_{m}$ shown in Fig. 4 are the semimajor axis and semiminor axis values which are given by

$$
\begin{aligned}
& a_{m}=\frac{c \tau_{m}}{2} \\
& b_{m}=\frac{1}{2} \sqrt{c^{2} \tau_{m}^{2}-D^{2}} .
\end{aligned}
$$

The ellipse shown in Fig. 4 may be described by either the Cartesian equation

$$
\frac{\left(x-\frac{D}{2}\right)^{2}}{a_{m}^{2}}+\frac{y^{2}}{b_{m}^{2}}=1
$$

or by the polar coordinate equation [9]

$$
r_{b}=\frac{c^{2} \tau_{m}^{2}-D^{2}}{2 c \tau_{m}-2 D \cos \left(\theta_{b}\right)}
$$

For each of the pdf's shown below, $D=1000 \mathrm{~m}$ and the maximum delay was $\tau_{m}=5 \mu \mathrm{s}$. Each of the histograms shown to validate the closed form expressions of the pdf are generated by placing 50000 scatterers uniformly into the ellipse, measuring the desired property (AOA or TOA), and then creating a histogram containing 75 bins. The number of points in each bin is divided by the total number of points to give the normalized histogram. In all cases, there is good agreement between the closed form expression and the normalized histogram.

\section{A. Joint TOA/AOA pdf (Elliptical Model)}

1) Joint TOA/AOA pdf at the Base Station: The joint TOA/ AOA pdf was derived in Section III for the case of a uniform scatterer density. The result given in (17) is repeated here for convenience

$$
f_{\tau, \theta_{b}}\left(\tau, \theta_{b}\right)=\frac{\left(D^{2}-\tau^{2} c^{2}\right)\left(D^{2} c+\tau^{2} c^{3}-2 \tau c^{2} D \cos \left(\theta_{b}\right)\right)}{4 A\left(D \cos \left(\theta_{b}\right)-\tau c\right)^{3}} .
$$

The area of an ellipse is given by $A=\pi a_{m} b_{m}$. Substituting this expression into (31) and explicitly stating the range of validity gives (32), shown at the bottom of the page. The case when $\theta_{b}=0$ is considered separately, due to the zero/zero condition which occurs when substituting $\theta_{b}=0$ and $\tau=$ $(D / c)$ directly into the first expression of (32). However, when $\theta_{b}=0$, several terms may be cancelled, leaving only

$$
\frac{c(D+\tau c)}{4 \pi a_{m} b_{m}}
$$

which may be evaluated at $\tau=(D / c)$.

For verification purposes, the analytically derived pdf's are compared against scatter plots. Fig. 5(a) and (b) shows the joint TOA/AOA pdf present at the base station for $D=1000$ $\mathrm{m}$ and $\tau_{m}=5 \mu \mathrm{s}$. Fig. 5(a) is a plot of the closed form expression for the joint pdf given in (32). Fig. 5(b) is a scatter plot created by randomly placing 10000 scatterers in the ellipse using a uniform distribution and then plotting the corresponding TOA/AOA pairs. The scatter plot shows a high concentration of points where the joint pdf is high. Also, both plots show a high concentration of scatterers with relatively small delays near the line-of-sight.

2) Joint TOA/AOA pdf at the Mobile: Due to the symmetry of the ellipse with respect to the base station and the mobile, by inspection, the same TOA/AOA pdf will be valid when related to the mobile perspective. The resulting joint pdf as a function of $\tau$ and $\theta_{s}$ is shown in (34) at the bottom of the next page.

\section{B. AOA pdf (Elliptical Model)}

1) AOA pdf at the Base Station: The polar equation defining the boundary of the scatterer region is

$$
r_{b}=\frac{\tau_{m}^{2} c^{2}-D^{2}}{2 \tau_{m} c-2 D \cos \left(\theta_{b}\right)}
$$

Since the base station is located inside the scatterer region, using (22), the AOA pdf is

$$
\begin{aligned}
f_{\theta_{b}}\left(\theta_{b}\right) & =\frac{1}{2 A} r_{b 2}^{2}\left(\theta_{b}\right) \\
& =\frac{1}{8 \pi a_{m} b_{m}}\left(\frac{\tau_{m}^{2} c^{2}-D^{2}}{\tau_{m} c-D \cos \left(\theta_{b}\right)}\right)^{2} .
\end{aligned}
$$

$$
f_{\tau, \theta_{b}}\left(\tau, \theta_{b}\right)= \begin{cases}\frac{\left(D^{2}-\tau^{2} c^{2}\right)\left(D^{2} c+\tau^{2} c^{3}-2 \tau c^{2} D \cos \left(\theta_{b}\right)\right)}{4 \pi a_{m} b_{m}\left(D \cos \left(\theta_{b}\right)-\tau c\right)^{3}}, & \frac{D}{c} \leq \tau \leq \tau_{m}, \quad \theta_{b} \neq 0 \\ \frac{c(D+\tau c)}{4 \pi a_{m} b_{m}}, & \frac{D}{c} \leq \tau \leq \tau_{m}, \quad \theta_{b}=0 \\ 0, & \text { else. }\end{cases}
$$




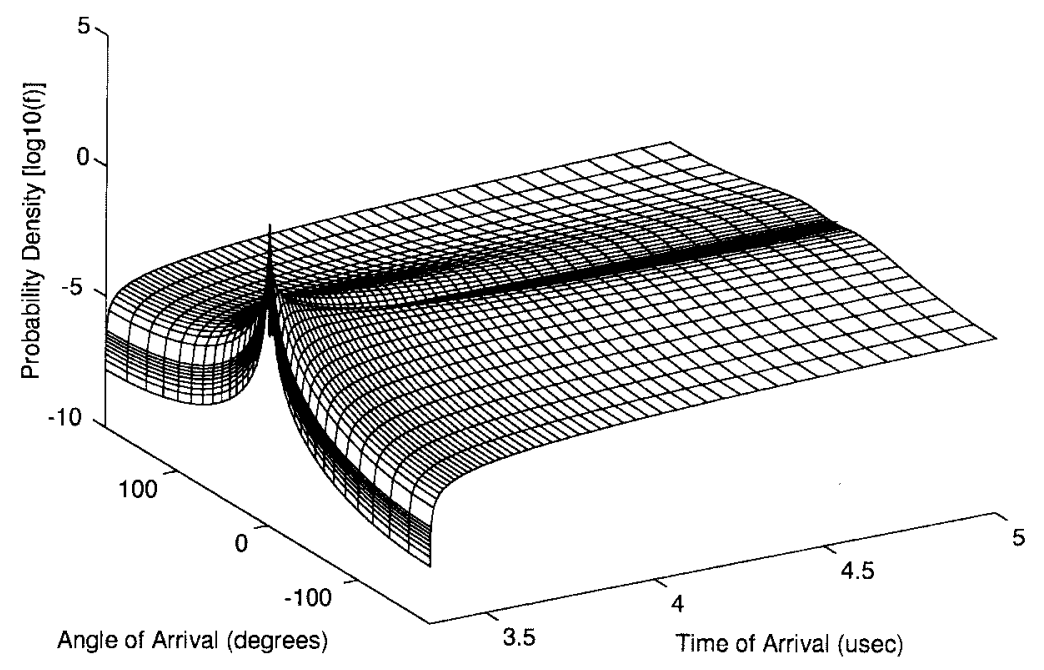

(a)

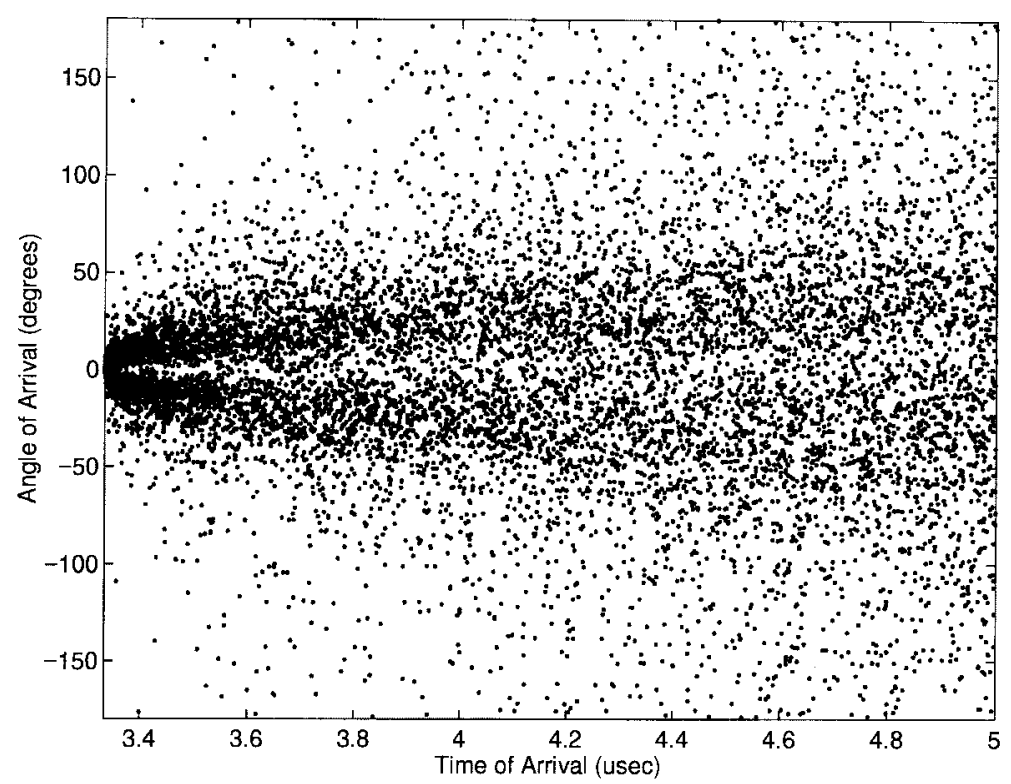

(b)

Fig. 5. Elliptical model: joint TOA/AOA pdf.

The elliptical AOA pdf and the normalized histogram are plotted in Fig. 6(a) for $D=1000 \mathrm{~m}$ and $\tau_{m}=5 \mu \mathrm{s}$. As expected from the joint TOA/AOA pdf, Fig. 6(a) shows that the scatterers are concentrated near line-of-sight.

2) AOA pdf at the Mobile: Again due to the symmetry of the ellipse, the same pdf applies at the mobile, namely

$$
\begin{aligned}
f_{\theta_{s}}\left(\theta_{s}\right) & =\frac{1}{2 A} r_{s 2}^{2}\left(\theta_{s}\right) \\
& =\frac{1}{8 \pi a_{m} b_{m}}\left(\frac{\tau_{m}^{2} c^{2}-D^{2}}{\tau_{m} c-D \cos \left(\theta_{s}\right)}\right)^{2}
\end{aligned}
$$

where $\theta_{s}=0$ in the direction toward the mobile and increases in a clockwise direction.

\section{TOA pdf (Elliptical Model)}

As derived earlier, the TOA pdf may be calculated using

$$
f_{\tau}(\tau)=\frac{1}{A} \frac{d}{d \tau}\left(A_{\tau}(\tau)\right)
$$

where $A_{\tau}(\tau)$ is the area of intersection of the ellipse corresponding to a delay of $\tau$ with the uniform scatterer region. For

$$
f_{\tau, \theta_{s}}\left(\tau, \theta_{s}\right)= \begin{cases}\frac{\left(D^{2}-\tau^{2} c^{2}\right)\left(D^{2} c+\tau^{2} c^{3}-2 \tau c^{2} D \cos \left(\theta_{s}\right)\right)}{4 \pi a_{m} b_{m}\left(D \cos \left(\theta_{s}\right)-\tau c\right)^{3}}, & \frac{D}{c} \leq \tau \leq \tau_{m}, \quad \theta_{s} \neq 0 \\ \frac{c(D+\tau c)}{4 \pi a_{m} b_{m}}, & \frac{D}{c} \leq \tau \leq \tau_{m}, \quad \theta_{s}=0 \\ 0, & \text { else. }\end{cases}
$$




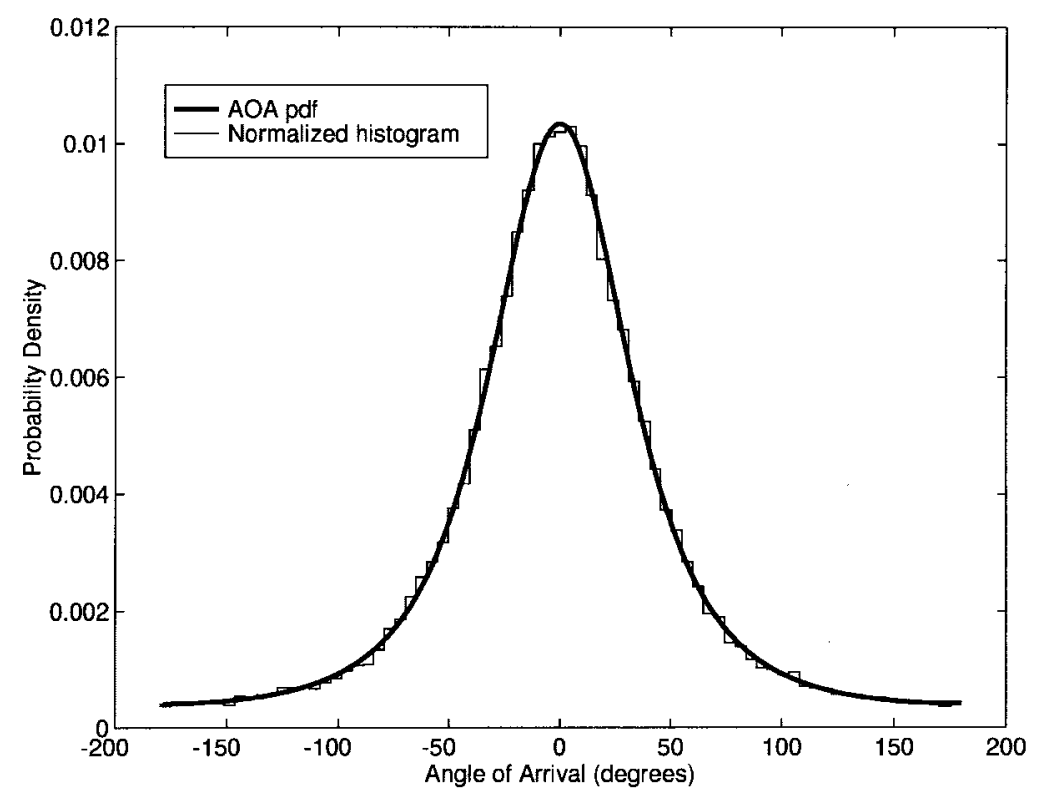

(a)

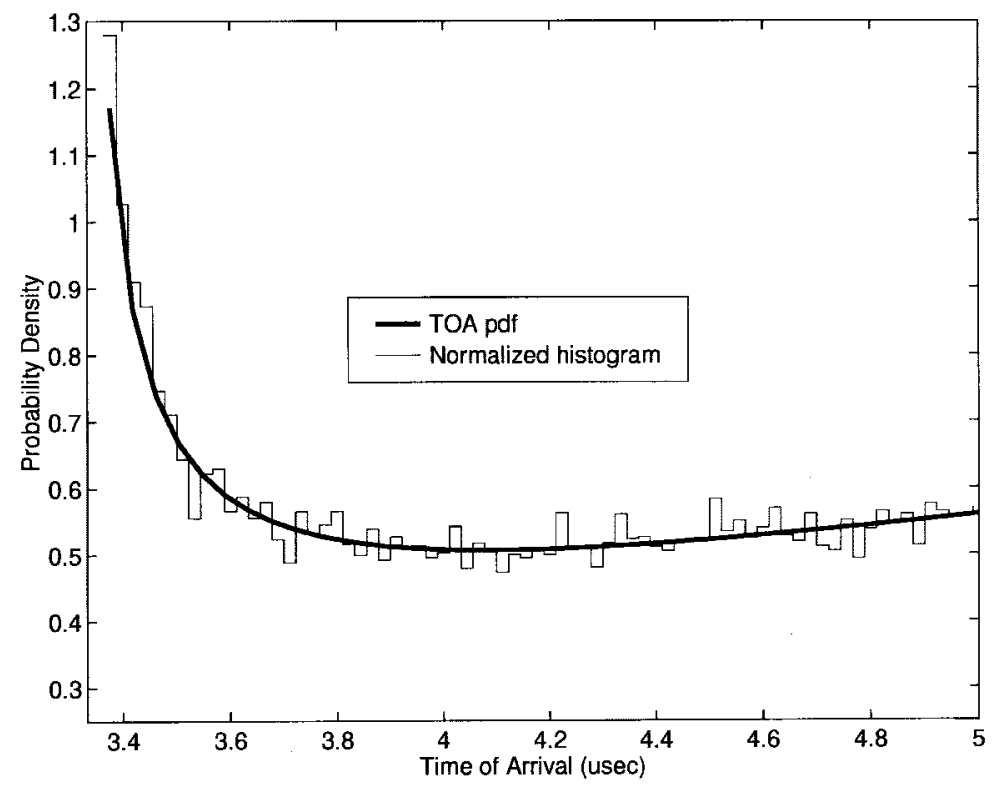

(b)

Fig. 6. Elliptical model: AOA and TOA pdf's.

the case of an elliptical scatterer region, $A_{\tau}(\tau)$ is the area of the ellipse itself. The area of an ellipse is given by

$$
A_{\tau}(\tau)=\pi a_{\tau} b_{\tau}=\frac{\pi \tau c}{v 4} \sqrt{\tau^{2} c^{2}-D^{2}}
$$

Taking the derivative with respect to $\tau$ and simplifying gives

$$
\frac{d}{d \tau}\left(A_{\tau}(\tau)\right)=\frac{\pi c\left(2 \tau^{2} c^{2}-D^{2}\right)}{4 \sqrt{\tau^{2} c^{2}-D^{2}}}
$$

Dividing by $A=\pi a_{m} b_{m}$ gives the TOA pdf

$$
f_{\tau}(\tau)= \begin{cases}\frac{c\left(2 \tau^{2} c^{2}-D^{2}\right)}{4 a_{m} b_{m} \sqrt{\tau^{2} c^{2}-D^{2}}}, & \frac{D}{c} \leq \tau \leq \tau_{m} \\ 0, & \text { else }\end{cases}
$$

Fig. 6(b) is a plot of the TOA pdf function and the normalized histogram. The plot shows that there is a high density of scatterers with relatively small delays.

\section{Circular Scattering Model}

The geometry of the circular scattering model (also referred to as the geometrically based single bounce macrocell model [1]) is shown in Fig. 7. The model assumes that the scatterers are uniformly distributed within a radius $R$ about the mobile. The model is based upon the assumption that in macrocell environments where antenna heights are relatively high, there will be no signal scattering from locations near the base station.

Although it is relatively easy to extend the pdf's derived below to allow any value of $R$, to maintain the premise of the 


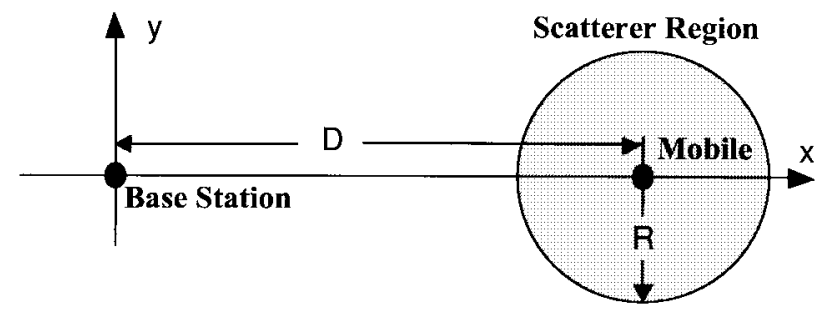

Fig. 7. Circular scatterer density geometry.

model that scatters are not located about the base station, we consider here only the case where $R<D$.

Each of the histograms shown to validate the closed form expressions of the pdf's were generated as described earlier for the elliptical model.

\section{A. Joint TOA/AOA pdf (Circular Model)}

1) Joint TOA/AOA pdf at the Base Station: When the scatterer region is circular, the area of the scatterer region is $A=\pi R^{2}$. Substituting this expression into (17) gives the joint TOA/AOA pdf shown in (42) at the bottom of the page. where the range of $\tau$ and $\theta_{b}$ for which $f_{\tau, \theta_{b}}\left(\tau, \theta_{b}\right) \neq 0$ was found by considering the condition on the original scatterer density, namely

$$
(x-D)^{2}+y^{2} \leq R^{2} .
$$

Expanding and then transforming into polar coordinates gives

$$
r_{b}^{2}-2 r_{b} D \cos \left(\theta_{b}\right)+D^{2} \leq R^{2} .
$$

Substituting the expression for $r_{b}$ found in (11) and then simplifying results in

$$
\frac{D^{2}-2 \tau c D \cos \left(\theta_{b}\right)+\tau^{2} c^{2}}{\tau c-D \cos \left(\theta_{b}\right)} \leq 2 R .
$$

Again, the case when $\theta_{b}=0$ is considered separately, due to the zero/zero condition which occurs when substituting $\theta_{b}=0$ and $\tau=(D / c)$ directly into the first expression of (42).

Fig. 8(a) and (b) shows the joint TOA/AOA pdf that is observed at the base station for $D=1000 \mathrm{~m}$ and $R=100 \mathrm{~m}$. Fig. 8(a) is a plot of the closed form expression given by (42).
Fig. 8(b) is a scatter plot created by placing 10000 scatterers and then plotting the corresponding TOA/AOA pairs.

2) Joint TOA/AOA pdf at the Mobile: As stated in Section III, the joint TOA/AOA pdf observed at the mobile will have the same form as the joint pdf observed at the base station with only the range of parameters where $f_{\tau, \theta_{b}}\left(\tau, \theta_{b}\right) \neq 0$ being different. The joint TOA/AOA pdf observed at the mobile is shown in (46) at the bottom of the page. The range of $\tau$ and $\theta_{s}$ for which $f_{\tau, \theta_{s}}\left(\tau, \theta_{s}\right) \neq 0$ is found by considering the condition on the original scatterer density, namely

$$
x_{c}^{2}+y^{2} \leq R^{2}
$$

where now for convenience the new Cartesian coordinate system $\left(x_{c}, y\right)$ is defined at the mobile such that $x_{c}=-x+D$. With this definition, the inequality given in (47) may be expressed as

$$
r_{s}^{2} \leq R^{2}
$$

where $r_{s}=\sqrt{x_{c}^{2}+y^{2}}$ is the radius of the polar coordinate system defined at the mobile. Substituting (18) into (48) gives the desired result

$$
\left(\frac{D^{2}-\tau^{2} c^{2}}{2\left(D \cos \left(\theta_{s}\right)-\tau c\right)}\right)^{2} \leq R^{2} .
$$

Fig. 8(c) and (d) show the joint TOA/AOA pdf observed at the mobile and a scatter plot of 10000 TOA/AOA samples from the circular model. There is good agreement between the two plots.

\section{B. AOA pdf (Circular Model)}

1) AOA pdf at the Base Station: The general expression for the AOA pdf was derived in Section IV-A. In this section, the AOA pdf corresponding to a circular scatterer density function is derived. The polar coordinate expression for a circle centered at $(0, D)$ with a radius $R$ is described by

$$
r_{b}^{2}-2 D \cos \left(\theta_{b}\right) r_{b}+D^{2}-R^{2}=0 .
$$

Using the quadratic formula

$$
r_{b}=D \cos \left(\theta_{b}\right) \pm \sqrt{D^{2} \cos ^{2}\left(\theta_{b}\right)-D^{2}+R^{2}}
$$

$$
f_{\tau, \theta_{b}}\left(\tau, \theta_{b}\right)= \begin{cases}\frac{\left(D^{2}-\tau^{2} c^{2}\right)\left(D^{2} c+\tau^{2} c^{3}-2 \tau c^{2} D \cos \left(\theta_{b}\right)\right)}{4 \pi R^{2}\left(D \cos \left(\theta_{b}\right)-\tau c\right)^{3}}, & \frac{D^{2}-2 \tau c D \cos \left(\theta_{b}\right)+\tau^{2} c^{2}}{\tau c-D \cos \left(\theta_{b}\right)} \leq 2 R, \theta_{b} \neq 0 \\ \frac{c(D+\tau c)}{4 \pi R^{2}}, & \frac{D}{c} \leq \tau \leq \frac{D+2 R}{c}, \\ 0, \quad \theta_{b}=0\end{cases}
$$

$$
f_{\tau, \theta_{s}}\left(\tau, \theta_{s}\right)= \begin{cases}\frac{\left(D^{2}-\tau^{2} c^{2}\right)\left(D^{2} c+\tau^{2} c^{3}-2 \tau c^{2} D \cos \left(\theta_{s}\right)\right)}{4 \pi R^{2}\left(D \cos \left(\theta_{s}\right)-\tau c\right)^{3}}, & \frac{D^{2}-\tau^{2} c^{2}}{2\left(D \cos \left(\theta_{s}\right)-\tau c\right)} \leq R, \quad \theta_{s} \neq 0 \\ \frac{c(D+\tau c)}{4 \pi R^{2}}, & \frac{D}{c} \leq \tau \leq \frac{D+2 R}{c}, \\ 0, \quad \theta_{s}=0\end{cases}
$$




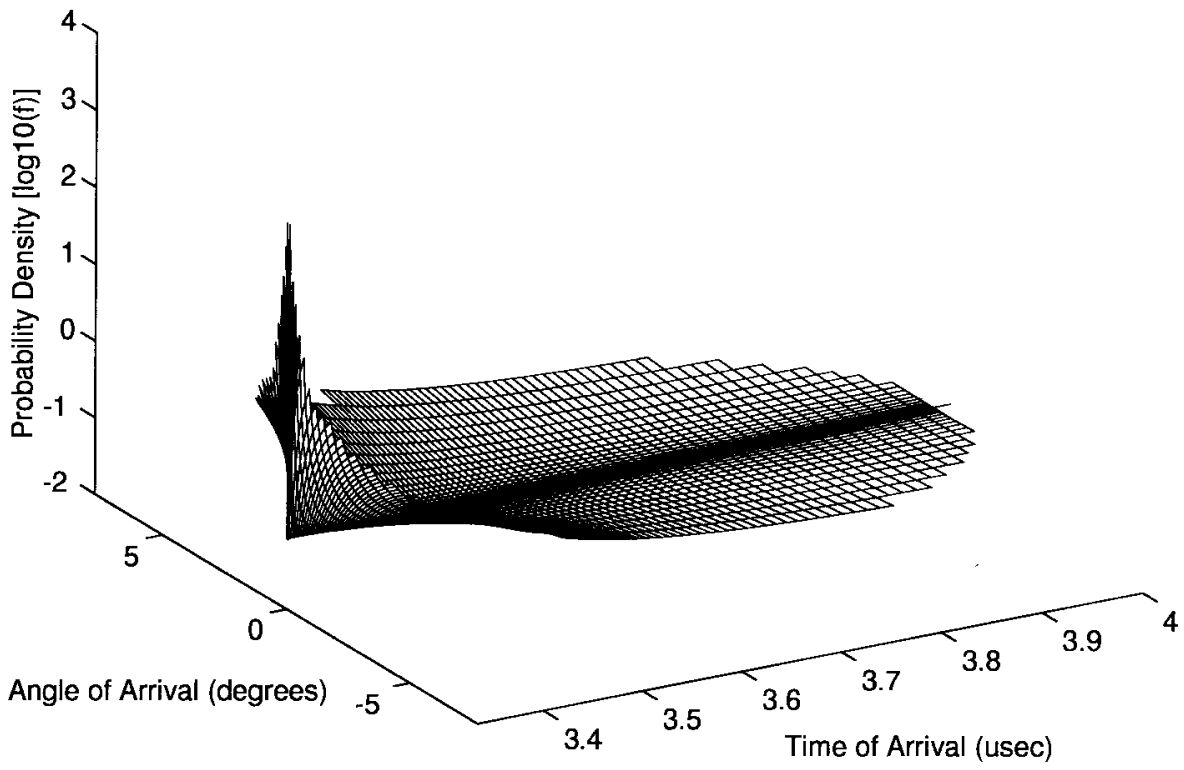

(a)

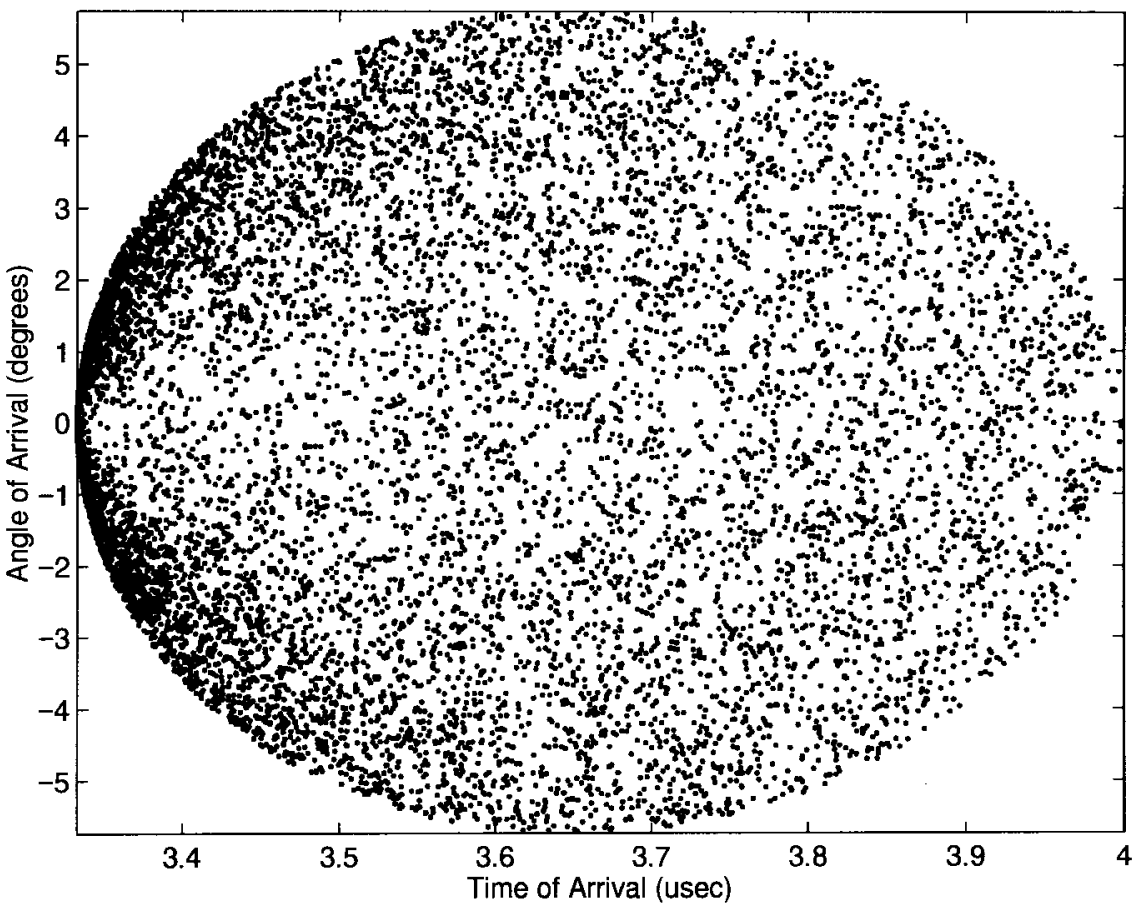

(b)

Fig. 8. Circular model: joint TOA/AOA pdf. (a) Joint pdf: base station (log-scale). (b) Scatter plot: base station.

which implies that

$$
\begin{aligned}
& r_{b 1}\left(\theta_{b}\right)=D \cos \left(\theta_{b}\right)-\sqrt{D^{2} \cos ^{2}\left(\theta_{b}\right)-D^{2}+R^{2}} \\
& r_{b 2}\left(\theta_{b}\right)=D \cos \left(\theta_{b}\right)+\sqrt{D^{2} \cos ^{2}\left(\theta_{b}\right)-D^{2}+R^{2}} .
\end{aligned}
$$

Substituting (52) and (53) into (21) with $A=\pi R^{2}$ and simplifying results in (54), shown at the bottom of the page.
Fig. 9 shows the AOA pdf observed at the base station for the case of $D=1000 \mathrm{~m}$ and $R=100 \mathrm{~m}$.

2) AOA pdf at the Mobile: The AOA pdf observed at the mobile is trivial. Since the scatterers are uniformly distributed within a radius about the mobile, the AOA density will be

$$
f_{\theta_{b}}\left(\theta_{b}\right)= \begin{cases}\frac{2 D \cos \left(\theta_{b}\right) \sqrt{D^{2} \cos ^{2}\left(\theta_{b}\right)-D^{2}+R^{2}}}{\pi R^{2}}, & \sin ^{-1}\left(\frac{R}{D}\right) \leq \theta_{b} \leq \sin ^{-1}\left(\frac{R}{D}\right) \\ 0, \text { else. } & \end{cases}
$$




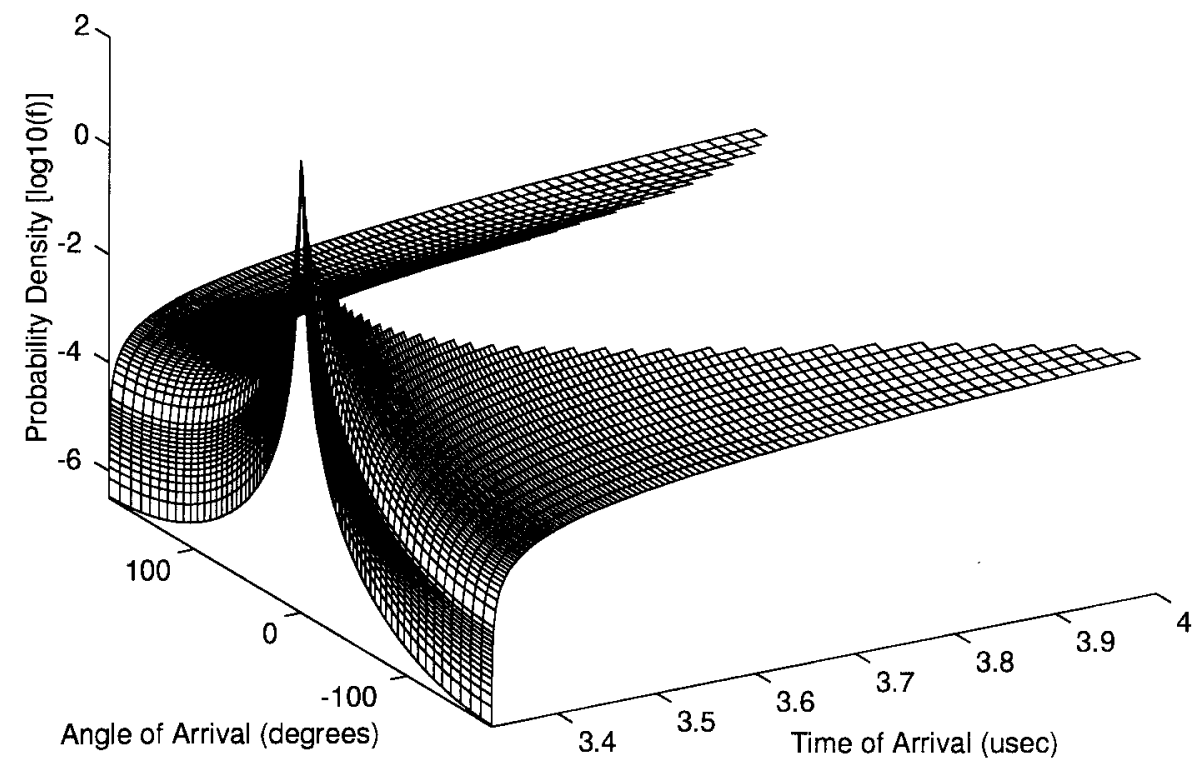

(c)

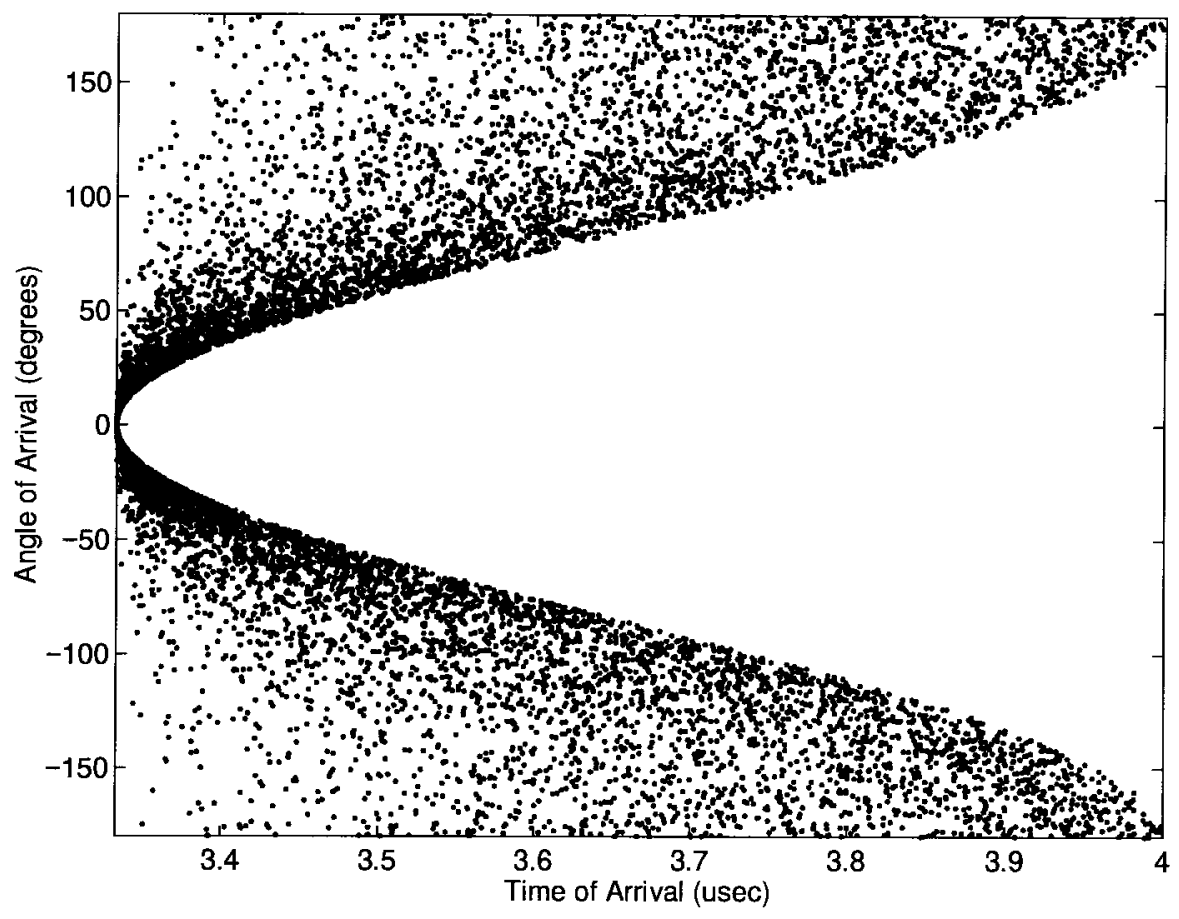

(d)

Fig. 8. (Continued). Circular model: joint TOA/AOA pdf. (c) Joint pdf: mobile (log-scale). (d) Scatter plot: mobile.

independent of angle. The resulting pdf is

$$
f_{\theta_{s}}\left(\theta_{s}\right)=\frac{1}{2 \pi}, \quad 0 \leq \theta_{s} \leq 2 \pi .
$$

\section{TOA pdf (Circular Model)}

The derivation of the TOA pdf, while straightforward, is tedious. The approach to be taken is to find the area of the intersection of an ellipse with major axis equal to $\tau c$ with the circular scatterer region. The derivative of the resulting function of $\tau$ divided by the total area of the circle gives the desired pdf (see Section IV-B)

$$
f_{\tau}(\tau)=\frac{1}{A} \frac{d}{d \tau}\left(A_{\tau}(\tau)\right)
$$

The area of intersection of the ellipse with the circular scatterer region is most easily derived using the polar coordinate equations of the circle and ellipse referred to the polar coordinate system defined at the mobile, $\left(r_{s}, \theta_{s}\right)$. (Note the nonconventional orientation of this coordinate system which is used for convenience, namely $\theta_{s}$ increases in the clockwise direction.) With respect to this coordinate system, the ellipse 


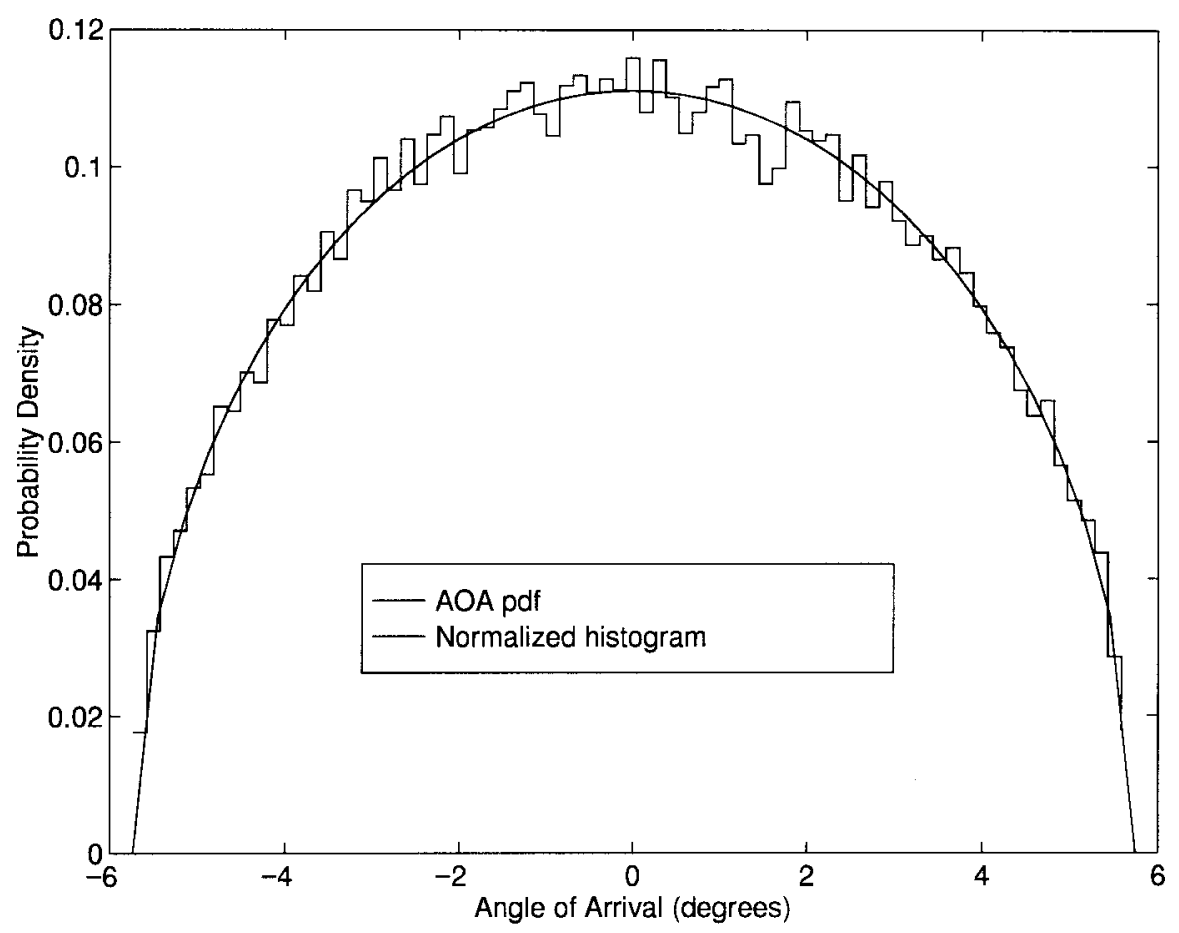

Fig. 9. Circular model: AOA pdf.

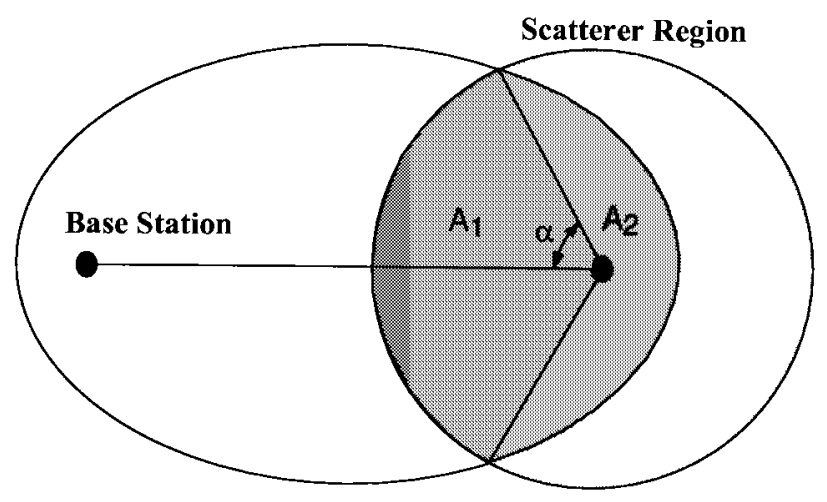

Fig. 10. Circular model: TOA pdf calculation.

and the circle are described by the following two equations, respectively

$$
\begin{aligned}
& r_{s_{e}}=\frac{c^{2} \tau^{2}-D^{2}}{2 c \tau-2 D \cos \left(\theta_{s}\right)} \\
& r_{s_{c}}=R
\end{aligned}
$$

Fig. 10 shows the situation being considered. The area of intersection is divided into two separate regions, $A_{1}$ and $A_{2}$, by the rays from the mobile to the points of intersection of the circle with the ellipse. The angle from the mobile to the point of intersection labeled $\alpha$ may be determined by substituting $\alpha$ for $\theta_{s}$ and solving (57) and (58) simultaneously for $\alpha$. The resulting positive value of $\alpha$ is

$$
\alpha=\operatorname{acos}\left(\frac{D^{2}+2 R \tau c-\tau^{2} c^{2}}{2 R D}\right)
$$

Since there is symmetry about the $x$ axis, it is possible to find the overall area of intersection by multiplying the area of intersection above the $x$ axis by two. Hence, the overall area of intersection may be expressed

$$
\begin{aligned}
A_{\tau}(\tau) & =A_{1}+A_{2} \\
& =2 \int_{0}^{\alpha} \frac{1}{2} r_{s_{c}}^{2}(\theta) d \theta+2 \int_{\alpha}^{\pi} \frac{1}{2} r_{s_{e}}^{2}(\theta) d \theta \\
& =\int_{0}^{\alpha} R^{2} d \theta+\int_{\alpha}^{\pi}\left(\frac{c^{2} \tau^{2}-D^{2}}{2 c \tau-2 D \cos (\theta)}\right)^{2} d \theta \\
& =R^{2} \alpha+\int_{\alpha}^{\pi}\left(\frac{c^{2} \tau^{2}-D^{2}}{2 c \tau-2 D \cos (\theta)}\right)^{2} d \theta .
\end{aligned}
$$

The last term may be integrated using integral formulas given in [10]. The resulting expression for the area as a function of $\tau$ becomes

$$
\begin{aligned}
A_{\tau}(\tau)= & R^{2} \alpha+\frac{D^{2}-\tau^{2} c^{2}}{4} \\
& \cdot\left[\frac{-\pi \tau c}{\sqrt{\tau^{2} c^{2}-D^{2}}}+\frac{D \sin (\alpha)}{\tau c-D \cos (\alpha)}+\frac{2 \tau c}{\sqrt{\tau^{2} c^{2}-D^{2}}}\right. \\
& \left.\cdot \operatorname{atan}\left(\frac{\sqrt{\tau^{2} c^{2}-D^{2}} \tan \left(\frac{\alpha}{2}\right)}{\tau c-D}\right)\right]
\end{aligned}
$$

Substituting the expression for $\alpha$ given in (59), taking the derivative with respect to $\tau$ with the aid of Matlab's Symbolic 


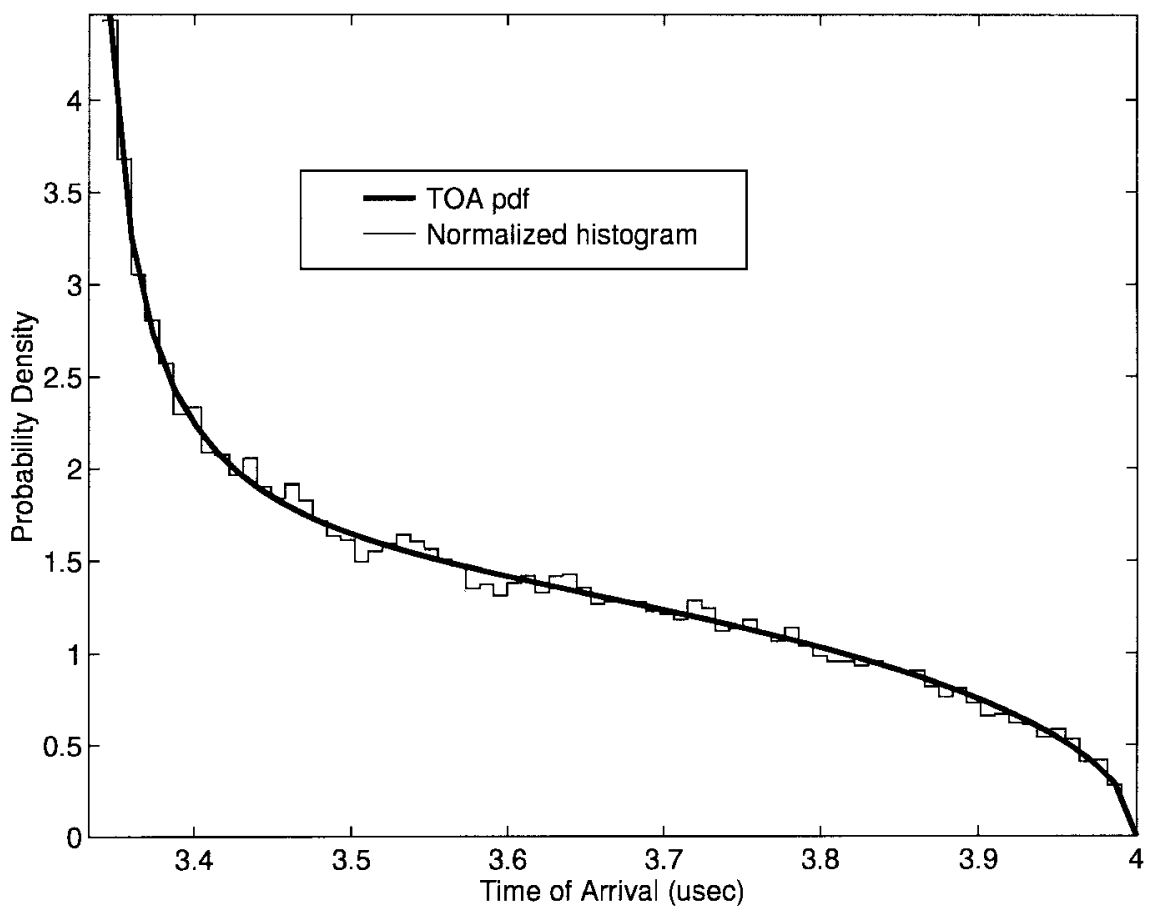

Fig. 11. Circular model: TOA pdf.

Toolbox, and then dividing by $A=\pi R^{2}$ gives the TOA pdf

$$
\begin{aligned}
f_{\tau}(\tau)= & \frac{c}{\pi R^{2}} \\
& \cdot\left[\frac{\pi \tau^{2} c^{2} k_{2}-\tau c k_{2}^{2}+\pi k_{2} k_{1}^{2}+\tau c k_{1}^{2}-2 R k_{1}^{2}}{4 k_{1} k_{2}}\right. \\
& +\frac{\tau^{2} c^{2} k_{0} k_{4}+\tau c k_{0} k_{1}^{2}}{2 k_{4}^{2}+2 k_{0}^{2} k_{1}^{2}}+\frac{\tau^{2} c^{2}+k_{1}^{2}}{2 k_{1}} \\
& \cdot \operatorname{atan}\left(\frac{k_{0} k_{1}}{k_{4}}\right)-\frac{R-\tau c}{\left(4 R^{2} D^{2}-k_{3}^{2}\right)^{1 / 2}} \\
& \left.\cdot\left(2 R^{2}+\frac{\tau c k_{1}^{2} k_{4}\left(1+k_{0}^{2}\right)}{2 k_{4}^{2}+2 k_{0}^{2} k_{1}^{2}}\right)\right]
\end{aligned}
$$

where

$$
\begin{aligned}
& k_{0}=\tan \left(\frac{1}{2} \operatorname{acos}\left(\frac{-\tau^{2} c^{2}+D^{2}+2 R \tau c}{2 R D}\right)\right) \\
& k_{1}=\sqrt{\tau^{2} c^{2}-D^{2}} \\
& k_{2}=\sqrt{D^{2}-4 R^{2}-\tau^{2} c^{2}+4 R \tau c} \\
& k_{3}=-\tau^{2} c^{2}+D^{2}+2 R \tau c \\
& k_{4}=D-\tau c .
\end{aligned}
$$

This expression is valid only for $(D / c)<\tau \leq(D+2 R / c)$. When $\tau=(D / c), k_{0}=0, k_{1}=0$, and $k_{4}=0$ and a few of the terms result in an indeterminate zero/zero condition. Although it may be possible to apply l'Hopital's rule and find the limit as $\tau \rightarrow(D / c)$, we will just restrict $\tau$ to be strictly greater than $D / c$. Fig. 11 shows the resulting TOA pdf and a simulated normalized histogram for $D=1000 \mathrm{~m}$ and $R=100$ $m$ verifying the validity of (65).

\section{CONCLUSIONS}

The joint TOA/AOA, marginal AOA, and marginal TOA pdf's for the elliptical and circular scattering models were derived. A general approach was taken that could easily be extended to other uniform scatterer density functions. General results were derived for the AOA marginal pdf's. Also an approach for deriving the TOA marginal pdf function was presented that could be applied to any scatterer density function.

\section{REFERENCES}

[1] P. Petrus, J. H. Reed, and T. S. Rappaport, "Geometrically based statistical channel model for macrocellular mobile environments," in Proc. IEEE GLOBECOM, 1996, pp. 1197-1201.

[2] J. C. Liberti and T. S. Rappaport, "A geometrically based model for lineof-sight multipath radio channels," in Proc. IEEE Vehicular Technology Conf., Apr. 1996, pp. 844-848.

[3] P. Zetterberg, P. Leth-Espensen, and P. Mogensen, "Propagation, beamsteering and uplink combining algorithms for cellular systems," presented at the ACTS Mobile Communications Summit, Royal Institute of Technology, Nov. 1996.

[4] J. Blanz, P. Jung, and P. Baier, "A flexibly configurable statistical channel model for mobile radio systems with directional diversity," in Proc. AGARD SSP Symp. Digital Communications Systems, 1995, pp. 38.1-10.

[5] M. Lu, T. Lo, and J. Litva, "A physical spatio-temporal model of multipath propagation channels," in Proc. IEEE Vehicular Technology Conf., 1997, pp. 810-814.

[6] O. Norklit and J. B. Andersen, "Mobile radio environments and adaptive arrays," PIMRC, 1994, pp. 725-728.

[7] J. C. Liberti, Jr. and T. S. Rappaport, Analysis of CDMA Cellular Radio Systems Employing Adaptive Antennas, Ph.D. dissertation, Virginia Polytechnic Institute and State Univ., Blacksburg, VA, Sept. 1995.

[8] A. Papoulis, Probability, Random Variables, and Stochastic Processes, 3rd ed. New York: McGraw-Hill, 1991. 
[9] G. B. Thomas, Jr. and R. L. Finney, Calculus and Analytic Geometry, 7th ed. New York: Addison-Wesley, 1988

[10] D. R. Lide, Ed., Handbook of Chemistry and Physics, 71st ed. Boston: CRC Press, Inc., 1990.

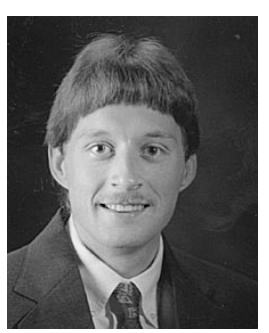

Richard B. Ertel was born on March 9, 1971, in Allentown, PA. He received the A.A. degree (with highest distinction) in engineering from Harrisburg Area Community College, Harrisburg, PA, in 1991, the B.S.E.E. degree (with highest honors) and the M.S.E.E. degree from Pennsylvania State University, State College, in 1993 and 1996, respectively, and the the Ph.D. degree in electrical engineering from Virginia Polytechnic and State University, Blacksburg, in 1999.

His work at Pennsylvania State University included a research assistantship at the Applied Research Lab in the summer of 1996, working on source localization. In the summer of 1997, he worked for Southwestern Bell Technology Resources as a Technical Intern evaluating and implementing vector channel models.

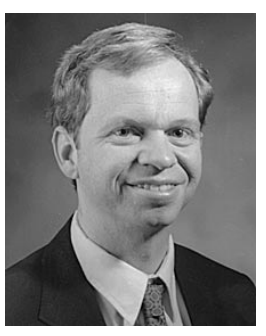

Jeffrey H. Reed (S'78-M'88-SM'98) received the B.S., M.S., and Ph.D. degrees in 1979, 1980, and 1987, respectively, from the University of California, Davis.

From 1980 to 1985, he was with Signal Science, Inc. in California and Maryland. From 1988 to 1992 , he served as a part-time Faculty Member at the University of California, Davis, and worked as a Consultant. In 1992, he joined the faculty at Virginia Polytechnic Institute and State University, Blacksburg, where he is an Associate Professor. He also serves as an Associate Director of the Mobile and Portable Radio Research Group and a Faculty Member of the Center for Wireless Telecommunications. His research interests are in software radios, location systems, interference rejection techniques, and adaptive array processing. 